\title{
SPACES ON WHICH EACH ABSOLUTELY SUMMING MAP IS NUCLEAR
}

\author{
D. R. LEWIS
}

\begin{abstract}
Let $E$ be a Banach space. The dual of $E$ is isometric to $l^{1}(\Gamma)$ for some set $\Gamma$ if and only if each absolutely summing operator on $E$ is nuclear, with equality of the nuclear and absolutely summing norms.
\end{abstract}

In [11] Stegall and Retherford have observed that each absolutely summing map on $c_{0}(\Gamma)$ is nuclear, and further have proven that this property isomorphically characterizes $c_{0}(\Gamma)$ among the spaces with unconditional bases. The object of this note is to show that this property, with the additional requirement that the nuclear and absolutely summing norms coincide, characterizes spaces with duals isometric to $l^{1}(\Gamma)$.

For $1 \leqq p \leqq \infty$ and $n$ a positive integer $l_{p}^{n}$ denotes the product of $n$ copies of the real numbers with the norm $\left\|\left(t_{1}, \cdots, t_{n}\right)\right\|=\left(\sum_{i \leqq n}\left|t_{i}\right|^{p}\right)^{1 / p}$ for $1 \leqq p<\infty$, and with the norm $\left\|\left(t_{1}, \cdots, t_{n}\right)\right\|=\max _{i \leqq n}\left|t_{i}\right|$ for $p=\infty$. For $E$ and $F$ two Banach spaces, the distance coefficient of $E$ and $F, d(E, F)$, is defined to be inf $\|u\|\left\|u^{-1}\right\|$, where the infimum is taken over all isomorphisms $u$ from $E$ onto $F$. For $1 \leqq p \leqq \infty$ and $1 \leqq \lambda$, a Banach space is termed a $\mathcal{L}_{p, \lambda}$-space if for each finite dimensional subspace $F \subset E$ there is a finite dimensional subspace $G$ with $F \subset G \subset E$ and $d\left(G, l_{p}^{n}\right) \leqq \lambda$, where $n$ is the dimension of $G$. The basic properties of the $\mathscr{L}_{p, \lambda}$-spaces may be found in [5], [6], and [11].

In regard to tensor products the notation and terminology is mainly that of [3]. In particular $\wedge$ denotes the greatest $\otimes$-norm, $\vee$ the least $\otimes$ norm and $\wedge$ the largest right injective $\otimes$-norm. An operator $u$ from $E$ into $F$ is said to be integral if and only if the bilinear form mapping $\left(x, y^{\prime}\right)$ to $\left\langle u(x), y^{\prime}\right\rangle$ induces, in a natural way, an element of $\left(E \stackrel{\otimes}{\otimes} F^{\prime}\right)^{\prime}$. The integral norm of such an operator is the norm of the induced functional and is written $\|u\|_{\wedge}$. An operator from $E$ into $F$ is nuclear if it is an image of an element of $E^{\prime} \hat{\otimes} F$ under the natural injection of $E^{\prime} \hat{\otimes} F$ into $L(E, F)$. In case $E^{\prime}$ has the approximation property the nuclear norm $u, N_{\wedge}(u)$, is the norm of its preimage in $E^{\prime} \hat{\otimes} F$. Following Pietsch [8] we say that an

Received by the editors February 16, 1971.

AMS 1970 subject classifications. Primary 46B99, 46B05.

Key words and phrases. Nuclear operators, absolutely summing operators, tensor products of Banach spaces. 
operator $u$ is absolutely summing if there is a constant $C$ such that $\sum_{i \leqq n}\left\|u\left(x_{i}\right)\right\| \leqq C \sup _{\left\|x^{\prime}\right\| \leqq 1} \sum_{i \leqq n}\left|\left\langle x_{i}, x^{\prime}\right\rangle\right|$ for every finite set $\left(x_{i}\right)$ in the domain of $u$. The absolutely summing norm of $u, \pi_{1}(u)$, is the smallest such constant. We note that an operator $u$ is absolutely summing if and only if it is of type $\wedge$, and that $\pi_{1}(u)=\|u\|_{\wedge}$ (Theorem 13 of [2] and Theorem 7 of [3]).

THEOREM. For a Banach space E, the following are equivalent:

(a) $E^{\prime}$ is isometric to $l^{1}(\Gamma)$ for some suitable set $\Gamma$.

(b) For every Banach space $F$, each absolutely summing $u: E \rightarrow F$ is nuclear and $\pi_{1}(u)=N_{\wedge}(u)$.

Proof. Assuming (a), $E$ must be $\mathcal{L}_{\infty, 1+\epsilon}$ for each $\epsilon>0$. The proof of Theorem III.3 of [11] shows that each absolutely summing operator on $E$ is integral and that the integral and absolutely summing norms are equal. Thus it is sufficient to show that an integral operator $u$ with integral norm 1 is nuclear and has nuclear norm at most 1 . Such a $u$ has a factorization $\beta \varphi \alpha$, where $\alpha: E \rightarrow L^{\infty}(\mu)$ and $\beta: L^{1}(\mu) \rightarrow F^{\prime \prime}$ have norm at most $1, \varphi$ is the injection of $L^{\infty}(\mu)$ into $L^{1}(\mu)$ and $(S, \Sigma, \mu)$ is a probability measure space.

Let $\lambda: \Sigma \rightarrow E^{\prime}$ be the measure given by $\lambda(A)=\alpha^{\prime}\left(\chi_{A}\right)$, where $L^{1}(\mu)$ is considered to be canonically embedded in $L^{\infty}(\mu)^{\prime}$. Then $\lambda$ is a $\mu$-continuous measure of total variation at most $\|\alpha\| \mu(S) \leqq 1$, so by Theorems 1 and 3 of [10] there is a Bochner integrable $g: S \rightarrow E^{\prime}$ such that $d \lambda=g d \mu$. Notice that for $A \in \Sigma, \int_{A} \alpha(x) d \mu=\langle x, \lambda(A)\rangle=\int_{A}\langle x, g\rangle d \mu$, so that $\alpha(x)=\langle x, g\rangle \mu$ a.e. More precisely, $\varphi \alpha(x)$ is the equivalence class in $L^{1}(\mu)$ containing $\langle x, g(\cdot)\rangle$. By Theorem 2 of [2], $\varphi \alpha$ is nuclear and so $\beta p \alpha: E \rightarrow F^{\prime \prime}$ is nuclear. The dual of $E$ has the approximation property so $u: E \rightarrow F$ is nuclear and $N_{\Lambda}(u) \leqq\|\beta\| N_{\Lambda}(\varphi \alpha) \leqq\|g\|_{1}=\vee(\lambda, S) \leqq 1$.

Assuming (b), each absolutely summing operator on $E$ is integral, so $E$ is a $\mathfrak{L}_{\infty}$-space [11, Theorem III.3] and hence $E^{\prime}$ is a $\mathfrak{L}_{1}$-space. A $\mathfrak{L}_{1}$-space which is a dual is complemented in some space $L^{1}(v)$ [15]. Since $L^{1}(v)$ has the approximation property, $E^{\prime}$ does also.

We first claim that for each Banach space $F$ the canonical injection $\alpha$ of $E^{\prime} \hat{\otimes} F^{\prime}$ into $E^{\prime} \hat{\otimes} F^{\prime}$ is an isometry onto. Obviously $\alpha$ has norm at most one and is one-to-one because $E^{\prime}$ has the approximation property. Let $\varphi \in E^{\prime} \hat{\otimes} F^{\prime}$ and consider $\varphi$ as an element of $B^{\wedge}(E, F)$. The operator $u: E \rightarrow F^{\prime}$ defined by $\langle y, u(x)\rangle=\langle x \otimes y, \varphi\rangle$ is of type $\wedge$ and $\|u\|_{\wedge}=$ $\|\varphi\|_{\wedge} \leqq|\varphi|_{\text {A }}$. By our hypothesis $u$ is nuclear so the bilinear form $\varphi$ is nuclear and $N_{\wedge}(\varphi)=N_{\wedge}(u)=\|u\|_{\wedge}$. Since $E^{\prime}$ has the approximation property there is a unique $\omega \in E^{\prime} \hat{\otimes} F^{\prime}$ which maps to $\varphi$ under the injection of $E^{\prime} \hat{\otimes} F^{\prime}$ into $B(E, F)$ and satisfies $|\omega|_{\wedge}=N_{\Lambda}(\varphi) \leqq|\varphi|_{\wedge}$. Clearly $\varphi=\alpha(\omega)$. 
Let $H$ be a Banach space, $G \subset H$ a closed subspace and consider the commutative diagram

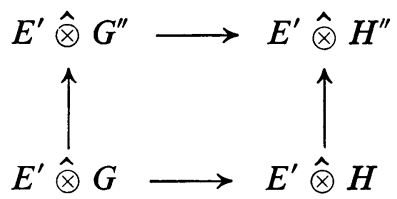

where each arrow indicates the canonical injection. By the preceding paragraph $E^{\prime} \hat{\otimes} G^{\prime \prime}=E^{\prime} \hat{\otimes} G^{\prime \prime}$ and $E^{\prime} \hat{\otimes} H^{\prime \prime}=E^{\prime} \hat{\otimes} H^{\prime \prime}$ isometrically. Since the $\otimes$-norm $\wedge$ is right injective, the top horizontal arrow is an into isometry. By Corollary 2, p. 13, of [3] each of the vertical arrows is isometric, we conclude that the injection of $E^{\prime} \hat{\otimes} G$ into $E^{\prime} \hat{\otimes} H$ is isometric. Since this is true whenever $G \subset H, E^{\prime}$ is isometric to a space $L^{1}(\mu)$ by [4]. It should be noted here that Stegall [12] has proven that $E^{\prime}$ is an $L^{1}(\mu)$ space assuming each absolutely summing $u: E \rightarrow F$ is integral with $\pi_{1}(u)=\|u\|_{A}$.

To complete the proof we need only show that $\mu$ is purely atomic. To this end we show that any set $A$ of positive finite $\mu$-measure contains a set of the same type which decomposes into a finite collection of atoms. Let $v$ be the restriction of $\mu$ to $A$ and $\lambda(B)$ be the functional in $E^{\prime}$ associated with $\chi_{A \cap B} \in L^{1}(\mu)=E^{\prime}$. Using the Radon-Nikodym theorem there is an operator $u: E \rightarrow L^{1}(v)$ satisfying $\left\langle u(x), \chi_{B}\right\rangle=\langle x, \lambda(B)\rangle$ for each $x \in E$ and measurable $B$. The operator maps the unit ball of $E$ into a lattice bounded set, so by Theorem 11 of [2] $u$ is integral. By (b) $u$ is nuclear and by Proposition 9 of [2] there is a $\nu$-Bochner integrable $g$ into $E^{\prime}$ such that $u(x)$ is the equivalence class in $L^{1}(v)$ containing $\langle x, g(\cdot)\rangle$. The function $g$ is a derivative of $\lambda$ with respect to $\mu$, so by Rieffel's theorem [9] there is a set $A_{0} \subset A$ of positive measure such that the closed circled convex hull, $K$, of the set of quotients $\mu(B)^{-1} \lambda(B), B \subset A_{0}$ and $\mu(B)>0$, is norm compact. This implies that the projection $p$ on $L^{1}(\mu)$ sending $f$ to $f \chi_{A_{0}}$ is compact. Indeed if $f=\sum_{i \leqq n} a_{i} \chi_{B_{i}}$ is simple (the $B_{i}$ 's disjoint) and $\|f\| \leqq 1$, then $p(f)$ is the sum

$$
\sum_{\mu\left(A_{0} \cap B_{i}\right)>0}\left[a_{i} \mu\left(A_{0} \cap B_{i}\right)\right]\left[\mu\left(A_{0} \cap B_{i}\right)^{-1} \lambda\left(A_{0} \cap B_{i}\right)\right]
$$

which is in the convex circled set $K$ because $\sum_{i}\left|a_{i}\right| \mu\left(A_{0} \cap B_{i}\right) \leqq\|f\| \leqq 1$. The simple functions of norm at most one are dense in the unit ball of $L^{1}(\mu)$, so $p$ maps the unit ball into $K$. If $n$ is the dimension of the range of $p$, then any partition of $A_{0}$ into sets of positive measure can be at most $n$ elements.

If $\mathfrak{T}$ is such a partition with the greatest possible number of elements, then each element of $\mathcal{T}$ is an atom. This completes the proof. 
It is known (see [1] and [7]) that for card $(\Gamma) \geqq \infty$ there are nonisomorphic spaces of continuous functions each of whose duals is isometric to $l^{1}(\Gamma)$. In particular, there are spaces not isomorphic to $c_{0}(\Gamma)$ which satisfy condition (b) of the theorem given above.

\section{BIBLIOGRAPHY}

1. C. Bessaga and A. Pełczyński, Spaces of continuous functions. IV. On isomorphical classification of spaces of continuous functions, Studia Math. 19 (1960), 53-62. MR 22 \#3971.

2. A. Grothendieck, Produits tensoriels topologiques et espaces nucléaires, Mem. Amer. Math. Soc. No. 16 (1955). MR 17, 763.

3. - Résumé de la théorie métrique des produits tensoriels topologiques, Bol. Soc. Math. São Paulo 8 (1953), 1-79. MR 20 \#1194.

4. - Une caractérisation vectorielle-métrique des espaces $L^{1}$, Canad. J. Math. 7 (1955), 552-561. MR 17, 877.

5. J. Lindenstrauss and A. Pełczyński, Absolutely summing operators in $L_{p}$-spaces and their applications, Studia Math. 29 (1968), 275-326. MR 37 \#6743.

6. J. Lindenstrauss and $H$. Rosenthal, The $\mathfrak{L}_{p}$-spaces, Israel J. Math. 7 (1969), 325-349.

7. A. Pełczyński and Z. Semadeni, Spaces of continuous functions. III. Spaces $C(\Omega)$ for $\Omega$ without perfect subsets, Studia Math. 18 (1959), 211-222. MR 21 \#6528.

8. A. Pietsch, Absolut p-summierende Abbildungen in normierten Raümen, Studia Math. 28 (1966/67), 333-353. MR 35 \#7162.

9. M. A. Rieffel, The Radon-Nikodým theorem for the Bochner integral, Trans. Amer. Math. Soc. 131 (1968), 466-487. MR 36 \#5297.

10. - Dentable subsets of Banach spaces, with application to a Radon-Nikodým theorem, Proc. Conference Functional Analysis (Irvine, Calif., 1966), Academic Press, London; Thompson, Washington, D.C., 1967, pp. 71-77. MR 36 \#5668.

11. C. P. Stegall and J. R. Retherford, Fully nuclear and completely nuclear operators with applications to $\mathfrak{L}_{1}$ and $\mathfrak{L}_{\infty}$-spaces, Trans. Amer. Math. Soc. (to appear).

12. C. P. Stegall, An operator characterization of spaces whose duals are $L^{1}(\mu)$, Israel J. Math. (to appear).

Department of Mathematics, Virginia Polytechnic Institute and State UniVERSITY, BLACKSBURG, VIRGINIA 24061 